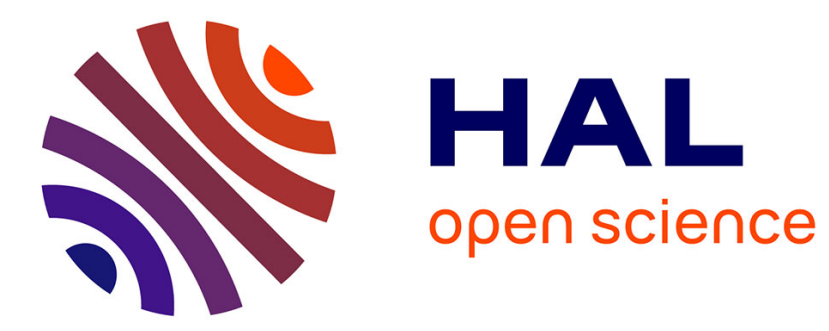

\title{
Subparticle stress fields in granular solids
}

Topin Vincent, Farhang Radjai, Jean-Yves Delenne

\section{To cite this version:}

Topin Vincent, Farhang Radjai, Jean-Yves Delenne. Subparticle stress fields in granular solids. Physical Review E : Statistical, Nonlinear, and Soft Matter Physics, 2009, 79 (5), pp.051302. 10.1103/PhysRevE.79.051302 . hal-00689882

\section{HAL Id: hal-00689882 https://hal.science/hal-00689882}

Submitted on 20 Apr 2012

HAL is a multi-disciplinary open access archive for the deposit and dissemination of scientific research documents, whether they are published or not. The documents may come from teaching and research institutions in France or abroad, or from public or private research centers.
L'archive ouverte pluridisciplinaire HAL, est destinée au dépôt et à la diffusion de documents scientifiques de niveau recherche, publiés ou non, émanant des établissements d'enseignement et de recherche français ou étrangers, des laboratoires publics ou privés. 


\title{
Sub-particle stress transmission in granular solids
}

\author{
Vincent Topin, Farhang Radjaï, and Jean-Yves Delenne \\ LMGC, CNRS-Université Montpellier II, Place Eugène Bataillon, 34095 Montpellier cedex, France
}

(Dated: February 3, 2009)

\begin{abstract}
We analyze stress transmission in granular media involving an interstitial cementing matrix of variable volume fraction. We rely on a lattice-type discretization of both the particles and cemented matrix. This Lattice Element Method gives access to elastic deformations and stress fields inside the particles and matrix, as well as at their interface. The signature of granular structure appears clearly on the probability density functions (pdf) of node stresses. We show that the stress chains are mainly guided by the particles in compression and by the matrix in tension. In tension, the stress component pdf's are increasingly broader for a decreasing matrix volume fraction whereas in compression they depend only on the particle stiffness. The pdf's are found to be gaussian inside the particles and exponential at their contact points. We compare in detail the contact force network computed from stresses localized at the matrix bridges between particles with that obtained from molecular dynamics simulations of the same system by using rigid particles with cohesive interactions. As far as contact forces are concerned the lattice element method yields the same force patterns and distributions as in molecular dynamics.
\end{abstract}

The granular microstructure is the common denominator of a broad class of solid materials including sedimentary rocks (sandstones, conglomerates and breccia)[3], some biomaterials such as wheat endosperm (starch granules forming a compact structure bound together by a protein matrix) [4-6], and many geomaterials like mortars, concrete and asphalt (aggregates of various sizes glued to each other by a cement paste) [1]. The presence of a particulate backbone endows these materials with similar failure properties.

Force distributions in dense granular media is at the focus of interests for granular mechanics, both from the viewpoint of mechanical processing and fondamental mechanisms at the microscopic scale. Severals experimental and numerical studies on dry granular media have shown that interparticles forces form a inhomogeneous contact network with an exponential pdf of the strong forces (REF???). However, most of it concerns only non cohesive granular materials, and cohesive binding between particles has been rarely taken into account (REF ???).

In this Letter, we are interested in stress transmission and force distribution in cemented granular materials (CGMs). CGMs form a wide class of materials with a similar microstructure consisting of densely packed particles and a solid matrix filling the interstitial space and sticking to the particles. Some examples are mortars, concrete and asphalt (aggregates of various sizes glued to each other by a cement paste) [1], solid propellants and high explosives (large volume of energetic particles in a polymeric binding matrix) [2], sedimentary rocks (sandstones, conglomerates and breccia) [3], and some biomaterials such as the wheat endosperm (starch granules form- ing a compact structure bound together by a protein matrix) $[4-6]$.

In the major numerical methods used to simulate granular materials (Contact Dynamics (CD), Discrete Element Method (DEM)), the particles are treated as solid objects that interact only at their contact points. The effect of the matrix can be represented as a cohesion law between particles, but volum effects due to the partial filling of interstitial spaces by the matrix cannot be considered easily. We rely here on a Lattice Element Method (LEM) based on the discretization of both the particles and cementing matrix. In this letter we analyse stress transmission in CGMs for the first time with a subparticle and interstial matrix resolution.

LEM has been extensively used for the statistical mechanics of fracture in disordered media [7], and applied to study the fracture properties of concrete [8], ceramics [9] and biomaterials like wheat endosperm $[5,6]$. The space is discretized as a grid of points (nodes) interconnected by one-dimensional elements (bonds). Each bond can transfer normal force, shear force and bending moment up to a threshold in force or energy. When several phases are present as in a cemented granular medium, each phase and its boundaries are materialized by lattice elements sharing the same properties and belonging to the same portion of space. For a cemented granular material, there are three bulk phases; the particles, the matrix and the voids, denoted respectively here 'p', 'm'; and 'v', and two kinds of interface phases; particle-particle interface, denoted 'pp', and particle-matrix interface, denoted 'pm'.

We use linear elastic-brittle elements, each element characterized by a Hooke constant and a breaking force threshold. The bonds transmit only normal forces between the lattice nodes and thus the strength of the lat- 
tice in shear and distortion is ensured only by the high connectivity of the nodes. A sample is defined by its contour and the configuration of the phases in space. The samples are deformed by imposing displacements or forces to nodes belonging to the contour. The initial state is the reference (unstressed) configuration. The total elastic energy of the system is a convex function of node displacements and thus finding the unique equilibrium configuration of the nodes amounts to a minimization problem. Performing this minimization for stepwise loading corresponds to subjecting the system to a quasistatic deformation process. The method used here can be found in more detail in reference see [10].

The cauchy stress tensor $\sigma$ makes sense only for a sufficiently large number of material points inside a control volume such that the surface density of forces is well defined. Following a general framework, first introduced by Moreau, we can attribute a stress tensor $\sigma^{i}$ to each node $i$ of the lattice network $[10,11]$. The physical content of this tensor remains the same whether applied to a node $i$ or to a portion of space including several nodes, and it tends to the Cauchy stress tensor at large scales. In order to analyze the stress distributions in our numerical samples, we use these "node stresses" whose components are represented by a proportional grey level over the elementary hexagonal cell centered on each node.

$$
\sigma_{\alpha \beta}^{i}=\frac{1}{V^{i}} \sum_{j} r_{\alpha}^{i j} f_{\beta}^{i j} .
$$

We first focus on the vertical stresses for a simple uniaxial compression test. We use a large dense packing of rigid disk-like particles compressed isotropically by means of discrete element method. A rectangular portion of this two-dimensional packing containing about 500 particles is then discretized on a triangular lattice. The matrix volume fraction $\rho^{m} \simeq 0$ here, corresponding to dry cohesive granular media.

Figure 1 shows the vertical stress fields $\sigma_{y y}$ at the undamaged state. The strong stress paths that we observe in this map are reminiscent of force chains observed both in cohesive and cohesionless granular media $[12,13]$. On figure 2 are represented forces between particles calculated by evaluating the total stress localized in the matrix bridges projected on the normal direction. The strong contact force network follows the stress chains observed before (fig. 1). The probability density function (pdf) of the vertical stresses is shown in Fig. 3. The stresses are normalized by the total stress $\sigma_{y y}$ of the lattice. From the shape of the pdf, we distinguish three parts : 1) Large stresses fall off exponentially as observed for large contact forces in dry granular media [12, 13]; 2) Weak stresses have nonzero probability reflecting the arching effect; 3 ) Intermediate stresses are centered on the mean and define a Gaussian distribution. Fig. ?? shows a typical map of vertical stresses where strong, weak and intermediate stresses are represented respectively in red, orange

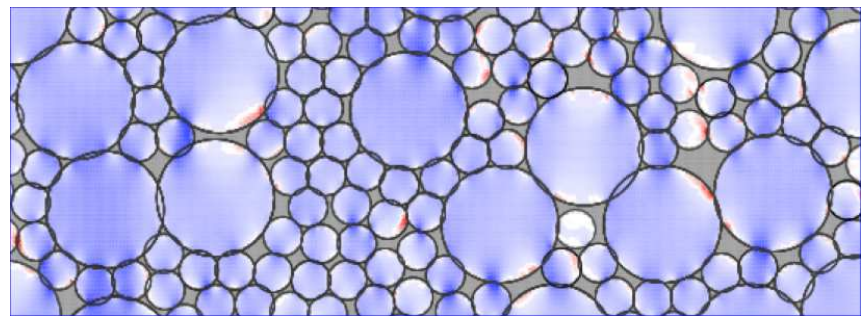

FIG. 1: Vertical stresses represented in levels of blue color.

(a)

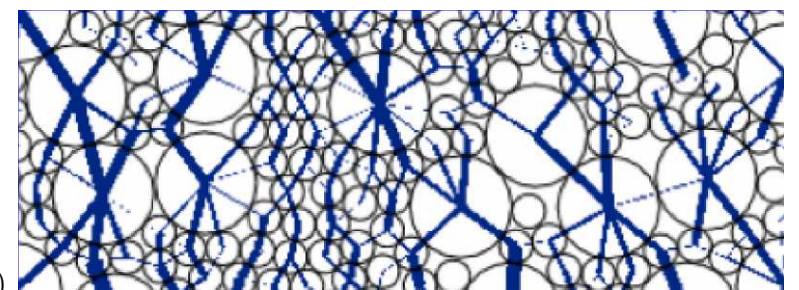

(b)

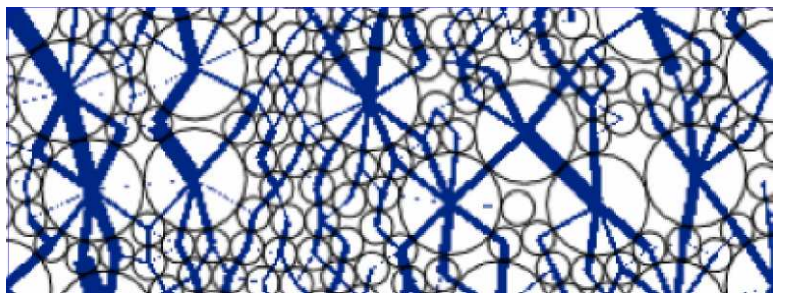

FIG. 2: Forces between particles calculated by evaluating the total stress localized in the matrix bridges projected on the normal direction.

and yellow. We see that the large stresses do mostly concentrate at the contact zones and they form well-defined chains that cross the particles. The intermediate stresses are almost fully localized inside the particles. Finally, weak stresses occurs at the surface zones or inside the unstressed particles.

We now consider the influence of loading for the same configuration of particles. The probablity density functions (pdf) in tension is plotted on the same fig. 3 in which is figured the pdf in compression. It is remarkable that, for the same matrix volume fraction, the distribution of large stresses is broader in compression than in tension. This means that stress distribution is far more inhomogeneous in compression than in tension. The range of large stresses corresponds to stress chains or, more precisely, to a "strong network" as in dry granular media where a well-defined subset of contact forces (the strong force network) transmits nearly the whole stress deviator sustained by the system. The effect of the matrix content is to redistribute more homogeneously the node stresses. Fig. 4 (a) and (b) show the pdf's of vertical stresses for different values of $\rho^{m}$ in tension and compression for a higher particle stiffness than matrix $\left(k^{p}=25 k^{m}\right)$. In tension, the pdf is increasingly wider for decreasing matrix content so that the stresses are more and more concen- 
[13] D. M. Mueth, H. M. Jaeger, and S. R. Nagel, Phys. 